\title{
ON THE ORIGIN OF ANISOTROPY IN MAGNETOHYDRODYNAMIC TURBULENCE: THE ROLE OF HIGHER-ORDER CORRELATIONS
}

\author{
Sean Oughton $^{1}$, MinPing Wan ${ }^{2}$, Sergio Servidio ${ }^{3}$, and William H. Matthaeus ${ }^{2}$ \\ ${ }^{1}$ Department of Mathematics, University of Waikato, Hamilton 3240, New Zealand \\ ${ }^{2}$ Bartol Research Institute and Department of Physics and Astronomy, University of Delaware, Newark, DE 19716, USA \\ ${ }^{3}$ Dipartimento di Fisica, Universita' della Calabria, I-87036 Cosenza, Italy \\ Received 2012 November 21; accepted 2013 February 6; published 2013 April 9
}

\begin{abstract}
Evolution of magnetohydrodynamic turbulence is often discussed in terms of second-order statistics like the energy spectra, but consideration of the structure of the von Kármán-Howarth hierarchy for MHD indicates that higherorder statistical correlations occupy an influential role. Here we show that both spectral anisotropy and energy decay are strongly associated with higher-order statistics. Dynamical emergence of spectral anisotropy must occur at a higher order in the statistical hierarchy, while numerical evidence suggests that strong variations in energy decay are connected with variations in higher-order statistics.
\end{abstract}

Key words: magnetic fields - magnetohydrodynamics (MHD) - turbulence

Online-only material: color figures

\section{INTRODUCTION}

A well-known feature of magnetohydrodynamics (MHD) is that a mean or average magnetic field induces a preferred direction that cannot be eliminated by a Galilean transformation. For MHD turbulence, an important consequence is the dynamical appearance of anisotropy in spectra and structure functions relative to the magnetic field. First seen in laboratory plasmas (Robinson \& Rusbridge 1971; Zweben et al. 1979) and later in the solar wind and corona (Matthaeus et al. 1990; Armstrong et al. 1990), this spectral anisotropy is of potential importance in a wide variety of astrophysical plasmas. For example, it has major influences on wave particle interactions and therefore on dissipation processes (Leamon et al. 2000) and scattering of energetic particles (Bieber et al. 1994).

The nature of the anisotropy is such that the gradients perpendicular to the magnetic field are greater than those measured parallel to it. This inequality is found whether the magnetic field direction is computed as a global mean or as a "local mean" (which can itself be computed in a variety of ways; Cho \& Vishniac 2000; Milano et al. 2001; Matthaeus et al. 2012). From the point of view of energy spectra, anisotropy implies that high wavenumber power is preferentially transferred into wavevectors that are in the perpendicular direction. This has motivated the development of dynamical models that postulate anisotropy as a premise, but do not explain its origin — reduced MHD (Kadomtsev \& Pogutse 1974; Strauss 1976; Montgomery 1982; Zank \& Matthaeus 1992), critical balance theory (Goldreich \& Sridhar 1995), and gyrokinetics (Schekochihin et al. 2009) are examples.

Explanations for spectral anisotropy are usually based on arguments related to weak turbulence, in which the dynamics are developed perturbatively beginning with propagating waves (Shebalin et al. 1983; Bondeson 1985; Grappin 1986; Oughton et al. 1994; Galtier et al. 2000, 2002; Schekochihin et al. 2012). While intuitively appealing, this picture addresses neither the statistical properties of turbulence that underlie spectral anisotropy nor its presence in strong turbulence. Here we discuss the origin of anisotropy in MHD turbulence beginning with the von Kármán-Howarth description, i.e., in terms of dynamical equations for correlations. We will show that while anisotropy has important consequences at the second-order level-e.g., for correlation functions and spectra-its origins are necessarily to be found in higher-order statistics. From this perspective it is apparent that anisotropy is closely connected with the higherorder statistics that control spectral transfer and energy decay.

Another issue related to higher-order correlations is the possible non-universality of MHD turbulence (Mininni 2011; Pouquet et al. 2011). Recent studies have reported strongly different evolution from ostensibly similar initial conditions (Lee et al. 2010). Here, we use simulations to show that this behavior is associated with significant variations in fourth-order correlations.

This paper is organized as follows. Orientation and physical motivation is provided in Section 2, after which we describe the origin of the von Kármán-Howarth equations in Section 3. In Section 4 we extend the discussion to emphasize the role a large-scale magnetic field plays in the development of anisotropy. Section 5 focuses on the influence of fourth-order correlations and presents some numerical examples. Conclusions are presented in Section 6, after which a short Appendix displays the full form of the equation for evolution of the thirdorder correlations.

\section{PHENOMENOLOGIES AT SECOND-ORDER}

We consider MHD turbulence that evolves in the presence of an externally supported mean magnetic field $\boldsymbol{B}_{0} \equiv B_{0} \hat{z}$. It is well known from laboratory experiments, solar wind observations, and simulations that a strong enough $\boldsymbol{B}_{0}$ induces anisotropy in the energy spectrum and alters the way in which energy decays (e.g., Robinson \& Rusbridge 1971; Zweben et al. 1979; Ren et al. 2011; Matthaeus et al. 1990; Bieber et al. 1996; Dasso et al. 2005; Horbury et al. 2008; Podesta 2009; Shebalin et al. 1983; Bondeson 1985; Grappin 1986; Carbone \& Veltri 1990; Oughton et al. 1994; Maron \& Goldreich 2001; Bigot et al. 2008a, 2008b). This occurs because nonlinear couplings are suppressed by Alfvén wave propagation effects, and these effects become stronger for interactions at shorter parallel wavelengths. Only couplings that do not change the parallel wavelengths are spared. 

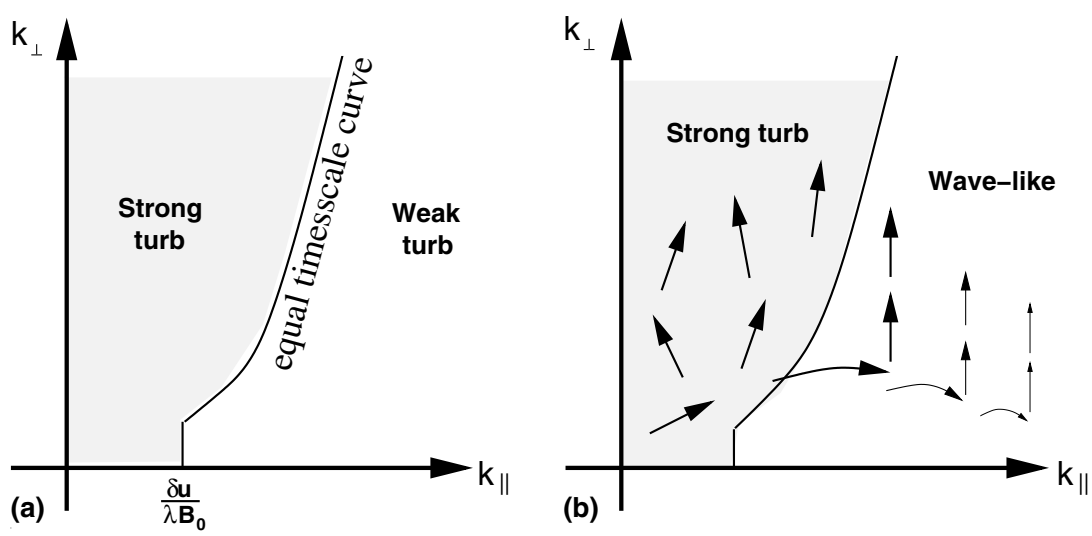

Figure 1. (a) Sketch of the Fourier space region where parallel transfer is suppressed ("weak turb"), due to the short wave timescales, and the unsuppressed region ("strong turb"), for which $\tau_{\mathrm{NL}}$ is faster than $\tau_{\mathrm{A}}$. The regions are separated roughly by the equal timescale curve, i.e., where the nonlinear timescale $\tau_{\mathrm{NL}}(\boldsymbol{k}) \approx 1 / k u_{k}$ and the wave timescale $\tau_{\mathrm{A}}=1 /\left|\boldsymbol{k} \cdot \boldsymbol{B}_{0}\right|$ are equal. (b) Similar sketch indicating some typical directions of energy transfer at example points. Note the dominantly perpendicular transfer for the weak turbulence regions and the approximately isotropic transfer inside the strong turbulence region.

Thus in regions of wavevector $(\boldsymbol{k})$ space in which propagation effects are strong, spectral transfer in the direction parallel to $\boldsymbol{B}_{0}$ (the "parallel direction") is relatively reduced, while perpendicular transfer is largely unchanged and thus tends to dominate (e.g., Galtier et al. 2000, 2002). The two Fourier space regions can be identified by comparing the nonlinear $\left(\tau_{\mathrm{NL}}\right)$ and wave $\left(\tau_{\mathrm{A}}\right)$ timescales at each $\boldsymbol{k}$-space position. Usually the faster timescale at each point will control the dynamics; see Figure 1.

Assuming these features of suppression and anisotropic spectral transfer, it is straightforward to build a dynamical description for the unaffected parts of wavenumber space based on the following scheme. We begin by adopting the phenomenological picture that the total energy transfer rate across a suitably defined surface in $\boldsymbol{k}$-space may be written in the inertial range as

$$
\epsilon=\frac{u_{k}^{2}}{\tau_{\mathrm{s}}},
$$

where $u_{k}^{2}$ is (proportional to) the energy per unit mass in fluctuations with scale size $1 / k$, and this expression serves to define the spectral transfer timescale $\tau_{\mathrm{s}}(\boldsymbol{k})$ at that same lengthscale, $1 / k$. In view of the implied relationship between $u_{k}^{2}$ and the omnidirectional energy spectrum $\mathcal{E}(k)$ [for isotropic turbulence $\left.\mathcal{E}(k) \sim u_{k}^{2} / k\right]$, it is apparent that if $\tau_{\mathrm{s}}$ could be expressed in terms of the spectrum, the problem would be closed at the level of second-order statistics.

If we further assume, following Kraichnan (1965), that $\epsilon$ should be directly proportional to the characteristic lifetime of third-order ("triple") correlations at scale $1 / k$, denoted $\tau_{3}(\boldsymbol{k})$, and that the only other available timescale is the Kolmogorov nonlinear ("eddy turnover") timescale $\tau_{\mathrm{NL}}(k)$, then one arrives at the relation $\tau_{\mathrm{s}} \tau_{3}=\tau_{\mathrm{NL}}^{2}$ (Kraichnan 1965; Pouquet et al. 1986; Matthaeus \& Zhou 1989; Zhou et al. 2004), sometimes known as the "golden rule." In this discussion the nonlinear time is assumed to be local in scale, but $\tau_{3}$, and therefore $\tau_{\mathrm{s}}$, may depend on the full wavevector $\boldsymbol{k}$ (not just its magnitude) and parametrically on other factors (e.g., $\boldsymbol{B}_{0}$, higher-order correlations) that need to be examined on a case by case basis. We write $\tau_{3}(*)$ to indicate this (so far) unspecified dependence. The parameterization of the spectral transfer rate then becomes

$$
\epsilon=\tau_{3}(*) \frac{u_{k}^{2}}{\tau_{\mathrm{NL}}^{2}(k)} .
$$

Many choices of $\tau_{3}$ are possible (e.g., Zhou et al. 2004). For example, if $\tau_{3}=\tau_{\mathrm{NL}}$, then isotropic Kolmogorov theory results. For $\tau_{3}=\left(k B_{0}\right)^{-1}$, the Iroshnikov-Kraichnan " $3 / 2$ " spectrum is found. ${ }^{4}$ If spectral transfer is assumed to be in the direction perpendicular to a mean magnetic field $\boldsymbol{B}_{0}$, then $k \rightarrow k_{\perp}$ and quasi-two-dimensional or two-dimensional turbulence can be described. When $\tau_{3}=\tau_{3}\left(k_{\|}\right)$but $\tau_{\mathrm{NL}}=\tau_{\mathrm{NL}}\left(k_{\perp}\right)$, then the weak turbulence form emerges.

This appealing, albeit phenomenological, approach can also be stated in terms of interaction of "wave packets" and is sufficiently powerful and revealing of turbulence properties that one can be led to believe that the problem is solved at the level of second-order correlations. Unfortunately this is not the case; the evolution of the spectrum ${ }^{5}$ is governed by the unclosed hierarchy of von Kármán-Howarth equations in which all orders of statistics appear.

\section{3. vON KÁRMÁN-HOWARTH EQUATIONS}

By considering homogeneous incompressible hydrodynamic turbulence, de Kármán \& Howarth (1938) showed that an infinite hierarchy of equations describes the time evolution of all two-point single-time correlation functions associated with the Navier-Stokes equation. The entire hierarchy is referred to as the von Kármán-Howarth (vKH) equations, although, a little confusingly, the equation for the second-order correlation is often called the vKH equation (Batchelor 1970; Monin \& Yaglom 1975).

Our focus is the MHD version of the vKH equations, again assuming homogeneity and incompressibility (Chandrasekhar 1951a, 1951b; Smith 1981; Politano \& Pouquet 1998a, 1998b; Wan et al. 2012). As the equations are well known, we provide below a simple sketch of their derivation. More detailed derivations are available elsewhere (e.g., Wan et al. 2012). Note that one can also obtain $\mathrm{vKH}$ equations for the structure functions, i.e., based on increments of the fluctuations (e.g., Politano \& Pouquet 1998b; MacBride et al. 2005, 2008; SorrisoValvo et al. 2007; Carbone et al. 2009).

It is convenient to employ Elsässer variables, denoted $z^{ \pm}=$ $\boldsymbol{v} \pm \boldsymbol{b}$. Here $v_{i}(\boldsymbol{x}, t)$ and $b_{i}(\boldsymbol{x}, t)$ are the Cartesian components

\footnotetext{
4 Note that this form for $\tau_{3}$ approximates $k_{\|}$as $k$, and thus essentially ignores the anisotropy of the Alfvén timescale: $\tau_{\mathrm{A}}=1 / k_{\|} B_{0}$.

5 Recall that the energy spectrum and the correlation function are Fourier transforms of each other (Batchelor 1970).
} 
of the solenoidal velocity and magnetic field fluctuations, the latter in Alfvén speed units. The mass density $\rho$ is assumed constant and uniform. A uniform DC magnetic field $\boldsymbol{B}_{0}=B_{0} \hat{\boldsymbol{z}}$ is included, and this is central to the discussion. The MHD equations take the form

$$
\partial_{t} z_{i}^{ \pm}=-\left(z_{k}^{\mp} \mp B_{0 k}\right) \partial_{k} z_{i}^{ \pm}-\partial_{i} P+v \partial_{k} \partial_{k} z_{i}^{ \pm},
$$

where $P$ is the total pressure (fluid plus magnetic) and $v$ is the kinematic viscosity, assumed equal to the resistivity for simplicity.

Using Equation (3) and its partner evaluated at the displaced position $\boldsymbol{x}^{\prime}=\boldsymbol{x}+\boldsymbol{r}$, instead of $\boldsymbol{x}$, it is straightforward to calculate the time derivative of the second-order correlation

$$
R^{ \pm}(\boldsymbol{r}, t)=\left\langle z^{ \pm} \cdot z^{ \pm \prime}\right\rangle
$$

The angle brackets indicate ensemble averaging and homogeneity requires that $R^{ \pm}$is a function of $\boldsymbol{r}=\boldsymbol{x}^{\prime}-\boldsymbol{x}$, but not of $\boldsymbol{x}$ or $\boldsymbol{x}^{\prime}{ }^{6}$ The shorthand notation $\boldsymbol{z}^{ \pm \prime} \equiv \boldsymbol{z}^{ \pm}\left(\boldsymbol{x}^{\prime}, t\right)$ is useful. Taking the time derivative yields the vKH equations for $R^{ \pm}$:

$$
\partial_{t} R^{ \pm}=-\frac{\partial}{\partial r_{k}}\left[\hat{Q}_{k}^{ \pm}(\boldsymbol{r})-\hat{Q}_{k}^{ \pm}(-\boldsymbol{r})\right]+2 v \frac{\partial^{2} R^{ \pm}}{\partial r_{k} \partial r_{k}}
$$

These clearly depend on the third-order correlations,

$$
\hat{Q}_{k}^{ \pm}(\boldsymbol{r}, t) \equiv\left\langle z_{k}^{\mp \prime} z^{ \pm} \cdot z^{ \pm \prime}\right\rangle,
$$

so that the second-order equations are not closed and thus an evolution equation for $\hat{Q}_{k}^{ \pm}(\boldsymbol{r}, t)$ is needed (see the Appendix). It is in turn not closed, depending upon several fourth-order correlations, for example, and so the hierarchy continues all the way to infinite order.

In obtaining Equation (5) spatial homogeneity is used, which implies $\left\langle z_{k}^{\mp} z^{ \pm} \cdot z^{ \pm \prime}\right\rangle=\hat{Q}_{k}^{ \pm}(-\boldsymbol{r}, t)$. Similar manipulations show that the pressure terms do not contribute at this order (Politano \& Pouquet 1998b), as is also the case for hydrodynamics (de Kármán \& Howarth 1938).

Since the $R^{ \pm}(\boldsymbol{r})$ are the inverse Fourier transforms of the Elsässer energy spectra, $E^{ \pm}(\boldsymbol{k})$, it follows that the vKH equations are completely equivalent to an evolution hierarchy for the spectra and their higher-order relatives. Thus, given the known anisotropy of the energy spectrum, one might anticipate that $\boldsymbol{B}_{0}$ would feature in Equation (5). Strikingly, it does not. So the question is, how can an equation that is ostensibly independent of $\boldsymbol{B}_{0}$ govern a correlation that shows $\boldsymbol{B}_{0}$-dependence? The situation is resolved by considering the higher-order vKH equations.

\section{STRUCTURE OF THE vKH EQUATIONS AND THE INFLUENCE OF $\boldsymbol{B}_{0}$}

As we have just noted, $\boldsymbol{B}_{0}$ does not feature explicitly in Equation (5), even though it is present in the MHD equation (3), in Equation (A1), and indeed all higher-order vKH equations. The second-order vKH equation is anomalous: it is the same for any value of $B_{0}$-including zero! This seems inconsistent with the well-established $\boldsymbol{B}_{0}$-induced anisotropy of $R^{ \pm}$, and also with the rate of energy decay being $\boldsymbol{B}_{0}$-dependent (e.g., Shebalin et al. 1983; Grappin 1986; Carbone \& Veltri 1990;

\footnotetext{
6 This is also true for all other homogeneous correlations.
}

Oughton et al. 1994; Bigot et al. 2008a, 2008b). In fact, there is no inconsistency because the third-order correlations $\hat{Q}_{k}^{ \pm}$do depend on $\boldsymbol{B}_{0}$, albeit implicitly. We now discuss the situation in detail.

Structurally, the equation for $Q_{k}^{ \pm}$contains several different types of terms (see the Appendix). Writing it and the secondorder vKH equation, Equation (5), schematically for a generic fluctuation $u$, we have

$$
\begin{gathered}
\partial_{t}\left\langle u u^{\prime}\right\rangle=\left\langle u u^{\prime} u^{\prime}\right\rangle+v\left\langle u u^{\prime}\right\rangle \\
\partial_{t}\left\langle u u^{\prime} u^{\prime}\right\rangle=\left\langle u u u^{\prime} u^{\prime}\right\rangle+B_{0 j}\left\langle u u^{\prime} \partial_{j} u^{\prime}\right\rangle+\left\langle P u u^{\prime}\right\rangle+v\left\langle u u^{\prime} u^{\prime}\right\rangle, \\
\partial_{t}\left\langle u u u^{\prime} u^{\prime}\right\rangle=\left\langle u u u^{\prime} u^{\prime} u^{\prime}\right\rangle+B_{0 j}\left\langle u u u^{\prime} \partial_{j} u^{\prime}\right\rangle+\ldots
\end{gathered}
$$

where for most terms spatial derivatives are not indicated. This skeletal form helps emphasize that the evolution of the third-order correlation depends explicitly on $\boldsymbol{B}_{0}$ (direction and magnitude), and hence so does its timescale $\tau_{3}\left(\boldsymbol{k}, \boldsymbol{B}_{0}\right)$. In other words, these two quantities will be anisotropic with respect to the direction of $\boldsymbol{B}_{0}$, even if they were initially isotropic. Recall that the $\boldsymbol{B}_{0}$-dependence of $\tau_{3}$ is a key feature in phenomenologies for the energy spectrum in MHD turbulence (e.g., Iroshnikov 1963; Kraichnan 1965; Montgomery 1982; Matthaeus \& Zhou 1989; Zhou \& Matthaeus 2005; Goldreich \& Sridhar 1995; Boldyrev 2006; Gogoberidze 2007).

In fact, the $\boldsymbol{B}_{0}$-dependence of $\hat{Q}^{ \pm} \sim\left\langle u u^{\prime} u^{\prime}\right\rangle$ is deeper than this. Since the fourth-order vKH equations depend explicitly on $\boldsymbol{B}_{0}$, the $\left\langle u u u^{\prime} u^{\prime}\right\rangle$ will have implicit $\boldsymbol{B}_{0}$-dependence, which will impact the evolution of $\left\langle u u^{\prime} u^{\prime}\right\rangle$. The structure is the same at higher orders so that the vKH hierarchy involves an infinite chain of such explicit/implicit $\boldsymbol{B}_{0}$ couplings. Note that the implicit effects of $\boldsymbol{B}_{0}$ are present at all orders, but in a subtle way since they "cascade in" from infinite order.

As far as anisotropy at second-order is concerned, there are several steps to the argument. The vKH equations mandate that it cannot begin at that order, since $\boldsymbol{B}_{0}$ does not appear in Equation (7). Thus, to generate anisotropy at secondorder, it must be that the third-order moment $\left\langle u u^{\prime} u^{\prime}\right\rangle$ is (or becomes) anisotropic. The discussion following Equation (9) shows that this will indeed be the case when $B_{0} \neq 0$. The anisotropy of $\left\langle u u^{\prime} u^{\prime}\right\rangle$ then feeds into Equation (7), engendering $\boldsymbol{B}_{0}$-dependence in $\left\langle u u^{\prime}\right\rangle$. This is the origin of anisotropy in the second-order correlations (including energy spectra).

The above discussion makes it clear that the lifetime of the triple correlations comes to depend upon $\boldsymbol{B}_{0}$, and this dependence influences the second-order correlations. Therefore, the entire influence of the mean magnetic field on the energy distribution - and upon spectral transfer-must proceed through its influence on the solutions of Equation (A1). We are led then to consider in greater detail the impact on turbulence structure and dynamics of the higher-order statistics, which we illustrate further in the following section.

\section{FOURTH-ORDER CORRELATIONS}

At this point it is instructive to present some numerical examples. These provide evidence of (1) how the initial values of fourth-order correlations are associated with distinct energy dynamics, even when $B_{0}=0$, and (2) how fourth-order correlations respond in runs with the same initial condition but different values of $B_{0}$. 


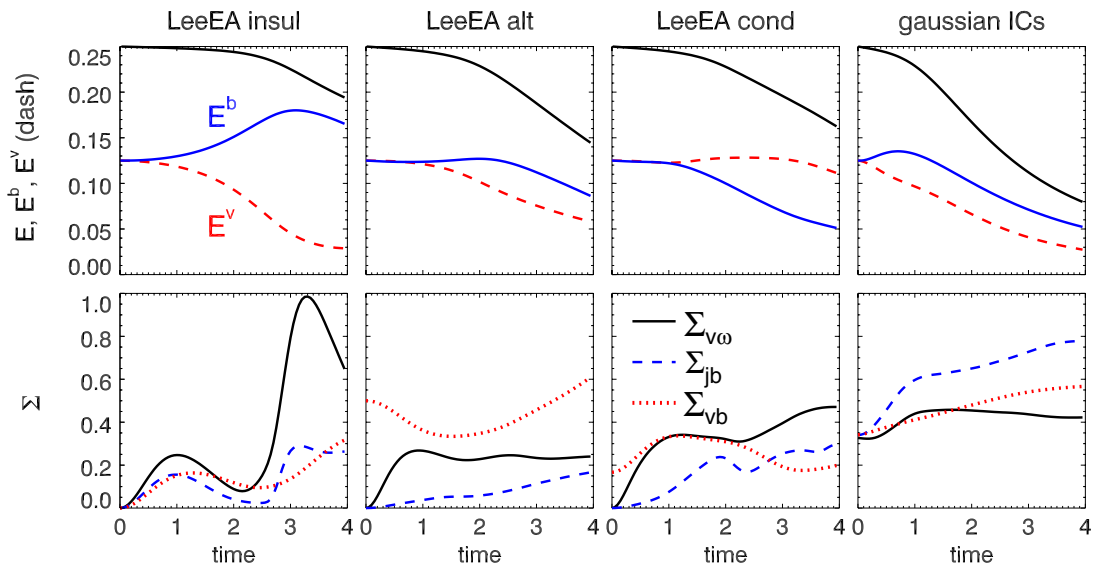

Figure 2. Some time histories from four $B_{0}=0$ runs with distinct initial conditions (see the main text). Each column corresponds to a different run. Top row: total, magnetic, and kinetic energies. Bottom row: normalized fourth-order moments of Equation (10).

(A color version of this figure is available in the online journal.)

The focus is on three normalized fourth-order quantities,

$$
\Sigma_{v \omega}=\frac{\left\langle(\boldsymbol{v} \cdot \boldsymbol{\omega})^{2}\right\rangle}{\left\langle\boldsymbol{v}^{2}\right\rangle\left\langle\boldsymbol{\omega}^{2}\right\rangle}, \quad \Sigma_{j b}=\frac{\left\langle(\boldsymbol{j} \cdot \boldsymbol{b})^{2}\right\rangle}{\left\langle\boldsymbol{j}^{2}\right\rangle\left\langle\boldsymbol{b}^{2}\right\rangle}, \quad \Sigma_{v b}=\frac{\left\langle(\boldsymbol{v} \cdot \boldsymbol{b})^{2}\right\rangle}{\left\langle\boldsymbol{v}^{2}\right\rangle\left\langle\boldsymbol{b}^{2}\right\rangle},
$$

where $\boldsymbol{\omega}=\nabla \times \boldsymbol{v}$ and $\boldsymbol{j}=\nabla \times \boldsymbol{b}$. Note that unlike the correlation functions considered in the previous sections, these are one-point correlations. Although there are many such, these ones are of particular interest because of their close relationship to local alignment properties of the three nonlinear terms in the "primitive" MHD equations: $\partial_{t} \boldsymbol{v} \sim \boldsymbol{v} \times \boldsymbol{\omega}+\boldsymbol{j} \times \boldsymbol{b}$ and $\partial_{t} \boldsymbol{b} \sim \nabla \times(\boldsymbol{v} \times \boldsymbol{b})$ (Servidio et al. 2008; Matthaeus et al. 2008). Consequently, one may interpret the $\Sigma s$ as a measure of the degree to which the appropriate fields are (locally) Beltrami, force-free, or Alfvénic, respectively.

\subsection{Runs with Different Initial $\boldsymbol{b}$ and $\Sigma_{v b}$}

The relationship between higher-order correlations and anisotropy is a major emphasis of this work. In this subsection, however, we consider four freely decaying $B_{0}=0$ runs, for which no global anisotropy is anticipated. Nonetheless, Equations (8)-(9) make it clear that $\tau_{3}$ is still influenced by higher-order correlations, and it is this aspect that we highlight here. In this subsection we also establish a context for the examination of the nonzero $B_{0}$ case that follows in Section 5.2.

The chosen runs each use a different initial condition (IC). Three of them start with the same (large-scale) Taylor-Green velocity field, but with distinct initial $\boldsymbol{b}$ fields. They are precisely the ICs used in Lee et al. (2010), where they are referred to as the "insulating," "conducting," and "alternative" runs. They are a convenient set to investigate in this context because $\Sigma_{v b}$ differs for each IC, but $\Sigma_{v \omega}$ and $\Sigma_{j b}$ are always initially zero. For each IC, the total energy is $E=0.25$, equally split between the kinetic $\left(E^{v}\right)$ and magnetic $\left(E^{b}\right)$ components, and the cross helicity and magnetic helicity are zero. The excited wavevectors are $\boldsymbol{k} \sim \pm(1,1,1)$ or $\boldsymbol{k} \sim \pm(2,2,2)$.

Summarizing, the Lee et al. runs each have a distinct $\boldsymbol{b}$ and $\Sigma_{v b}$, but most other familiar parameters are identical or very similar. Their evolution is compared with a fourth run, which starts from a broadband state with Gaussian random phase fluctuations in the wavenumber range $3 \leqslant k \leqslant 6$. The initial energies match those of the Lee et al. (2010) runs:
$E^{v}=E^{b}=0.125$. The magnetic helicity and cross helicity are both small for this run.

On the numerical details side, a Fourier pseudospectral code with second-order Runge-Kutta timestepping is employed. The resolution is $512^{3}$, with $v=1.1 \times 10^{-3}$ (equal to the resistivity). This combination ensures that the cutoff wavenumber $\left(k_{\text {wall }}=N / 2=256\right)$ is at least triple the maximum Kolmogorov dissipation wavenumber, a criterion that is important for obtaining accurate higher-order statistics, like the $\Sigma$ s (e.g., Wan et al. 2010; Donzis et al. 2008).

Figure 2 displays the evolution of the energies for each run. This is as reported in Lee et al. (2010), where implications for non-universality of MHD turbulence are discussed. As they remarked, the "conducting" run is anomalous compared to the others, in that $E^{v}$ exceeds $E^{b}$. The other three runs have qualitatively similar behaviors, with the magnetic energy predominant, and (not shown) the enstrophy $\left\langle\boldsymbol{\omega}^{2}\right\rangle,\left\langle\boldsymbol{j}^{2}\right\rangle$, and kurtoses having a single peak, with the magnetic quantities being larger. There are, however, some significant quantitative differences (cf. the spectra plots in Lee et al. 2010). Specifically, the decay rates and the $E^{v} / E^{b}$ ratio vary markedly across the runs, prompting concerns over the universality of MHD turbulence (e.g., Lee et al. 2010; Mininni 2011; Pouquet et al. 2011).

The $\Sigma$ time histories are also shown in Figure 2. Clearly there are similarities and differences between the runs, and one should be careful about drawing conclusions from such a small set of examples. Still, each $\Sigma$ tends to grow, although often not monotonically. There is also an indication that extrema of the $\Sigma$ s are correlated with peaks in familiar quantities like the mean-square current and the enstrophy (not shown; cf. Oughton et al. 2013). It may prove to be difficult to elicit details of how higher-order correlations like the $\Sigma$ s influence the flow dynamics. Nonetheless, these examples suggest that the $\Sigma$ s may well be parameters of importance to the dynamics and long-time states. $^{7}$

\subsection{Influence of $B_{0}$ on the $\Sigma$}

For this investigation, we performed three $512^{3}$ runs with $v=2.4 \times 10^{-4}$ and the same IC, constructed using Gaussian random phases for Fourier modes with $3 \leqslant k \leqslant 8$. The value of

\footnotetext{
7 See Wan et al. (2012) for a discussion connecting universality in MHD turbulence and the $\Sigma$ s.
} 

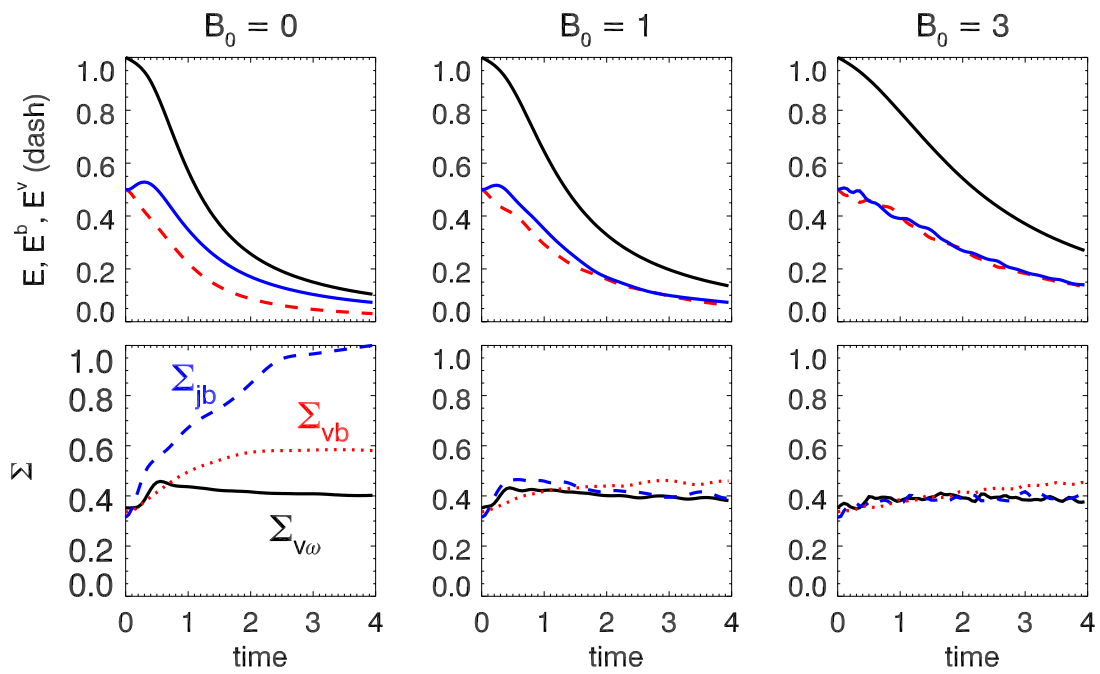

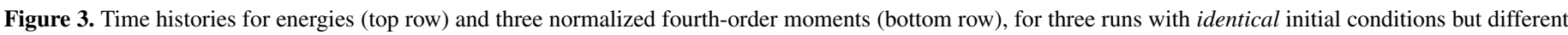
mean field strengths, $B_{0}$. Each column corresponds to a different run.

(A color version of this figure is available in the online journal.)

$v$ (equal to the resistivity) is again chosen to ensure the runs are well resolved. The only initial quantity that differs for the runs is the value of $B_{0}=0,1,3$.

The well-known reduction of the energy decay rate is in evidence (Figure 3, top row), and similarly the stronger equipartitioning of the kinetic and magnetic energy with increasing $B_{0}$ (e.g., Oughton et al. 1994; Bigot et al. 2008b). The new result is that $B_{0}$ acts to strongly curtail the growth of $\Sigma_{j b}$, and to a lesser extent, the growth of $\Sigma_{v b}$. This suggests that it is suppression of these fourth-order correlations that is associated with the development of anisotropy. For the $B_{0} \neq 0$ cases, there may also be a connection with the non-conservation of magnetic helicity (e.g., Matthaeus \& Goldstein 1982; Stribling et al. 1994; Blackman \& Field 2000; Cho et al. 2009). At least for these ICs, the non-magnetic $\Sigma_{v \omega}$ is not strongly affected by the strength of $B_{0}$.

\section{CONCLUSIONS}

We have highlighted how the absence of the DC magnetic field from the second-order vKH equation is really only an apparent absence since the unclosed nature of the equation supports an implicit dependence on $\boldsymbol{B}_{0}$ through the third-order correlations. From the structure of the hierarchy of equations for incompressible homogeneous MHD turbulence, it is evident that anisotropy arises solely due to higher-order statistical effects, and that the appearance of anisotropy in second-order statistical quantities such as spectra and associated correlation functions depends entirely on mean magnetic field effects that occur in these higher-order equations.

The $\boldsymbol{B}_{0}$-dependence of the energy spectrum has been used in physical phenomenologies of the spectrum for many decades, starting with the Iroshnikov (1963) and Kraichnan (1965) models. However, the formal mathematical arguments justifying it do not appear to be well known, as many theoretical works rely on either one-point closure arguments or weak turbulence arguments, and in these approaches the point we emphasize here is not treated prominently. The origin of anisotropy becomes clearer from consideration of the vKH hierarchy and its unclosed nature.
Furthermore, recognition of the origin of anisotropy in higherorder statistics sheds light on the well-documented enhancements of anisotropy that are seen in local statistics (which are necessarily of higher order; Matthaeus et al. 2012). Such considerations motivate additional study of non-Gaussian effects and intermittency that influences the structure of the fourth- and higher-order statistical quantities in the vKH hierarchy.

We have also presented here several numerical examples revealing the correspondence between different (kinetic and magnetic) energy decay rates and several fourth-order correlations. In this regard, the details of cause and effect remain to be determined, as do the connections between fourth-order correlations and the seeming non-universality of MHD turbulence.

This research is supported in part by the NSF Solar Terrestrial Program Grant AGS-1063439 and SHINE grant AGS-1156094, NASA Heliophysics Theory Program, NNX11AJ44G, POR Calabria FSE-2007/2013, and by the EU Turboplasmas project.

\section{APPENDIX}

\section{THE THIRD-ORDER EVOLUTION EQUATION}

For $Q_{k}^{ \pm}(\boldsymbol{r}, t)$, this takes the form,

$$
\begin{aligned}
\partial_{t} \hat{Q}_{k}^{ \pm}= & \frac{\partial}{\partial r_{m}}\left\langle z_{m}^{\mp} z_{k}^{\mp \prime} z^{ \pm} \cdot z^{ \pm \prime}\right\rangle-\left\langle z_{k}^{\mp \prime} \partial_{m}^{\prime}\left(z_{m}^{\mp \prime} z^{ \pm} \cdot z^{ \pm \prime}\right)\right\rangle \\
& -\left\langle z^{ \pm} \cdot z^{ \pm \prime} \partial_{m}^{\prime}\left(z_{m}^{ \pm \prime} z_{k}^{\mp \prime}\right)\right\rangle \mp 2 B_{0 m}\left\langle z^{ \pm} \cdot z^{ \pm \prime} \partial_{m}^{\prime} z_{k}^{\mp \prime}\right\rangle \\
& +\frac{\partial}{\partial r_{i}}\left\langle P z_{i}^{ \pm \prime} z_{k}^{\mp \prime}\right\rangle-\left\langle z_{k}^{\mp \prime} \partial_{i}^{\prime}\left(P^{\prime} z_{i}^{ \pm}\right)\right\rangle-\left\langle z^{ \pm} \cdot z^{ \pm \prime} \partial_{k}^{\prime} P^{\prime}\right\rangle \\
& +v \frac{\partial^{2}}{\partial r_{m}^{2}}\left\langle z_{k}^{\mp \prime} z^{ \pm} \cdot z^{ \pm \prime}\right\rangle+v\left\langle z_{k}^{\mp \prime} \partial_{m}^{\prime} \partial_{m}^{\prime}\left(z^{ \pm} \cdot z^{ \pm \prime}\right)\right\rangle \\
& +v\left\langle z^{ \pm} \cdot z^{ \pm \prime} \partial_{m}^{\prime} \partial_{m}^{\prime} z_{k}^{\mp \prime}\right\rangle,
\end{aligned}
$$

with $\partial_{m}^{\prime}=\partial / \partial x_{m}^{\prime}$. The first three terms are all fourth-order correlations, in which $B_{0}$ does not appear (explicitly). There is precisely one term with $\boldsymbol{B}_{0}$ in it, contracted with a third-order correlation. The remaining terms are third-order correlations involving the pressure, and three further third-order correlations multiplied by the viscosity. 


\section{REFERENCES}

Armstrong, J., Coles, W., Kojima, M., \& Rickett, B. 1990, ApJ, 358, 685

Batchelor, G. K. 1970, The Theory of Homogeneous Turbulence (Cambridge: Cambridge Univ. Press)

Bieber, J. W., Matthaeus, W. H., Smith, C. W., et al. 1994, ApJ, 420, 294

Bieber, J. W., Wanner, W., \& Matthaeus, W. H. 1996, JGR, 101, 2511

Bigot, B., Galtier, S., \& Politano, H. 2008a, PhRvE, 78, 066301

Bigot, B., Galtier, S., \& Politano, H. 2008b, PhRvL, 100, 074502

Blackman, E. G., \& Field, G. B. 2000, ApJ, 534, 984

Boldyrev, S. 2006, PhRvL, 96, 115002

Bondeson, A. 1985, PhFl, 28, 2406

Carbone, V., Sorriso-Valvo, L., \& Marino, R. 2009, EL, 88, 25001

Carbone, V., \& Veltri, P. 1990, GApFD, 52, 153

Chandrasekhar, S. 1951a, RSPSA, 204, 435

Chandrasekhar, S. 1951b, RSPSA, 207, 301

Cho, J., \& Vishniac, E. T. 2000, ApJ, 539, 273

Cho, J., Vishniac, E. T., Beresnyak, A., Lazarian, A., \& Ryu, D. 2009, ApJ, 693, 1449

Dasso, S., Milano, L. J., Matthaeus, W. H., \& Smith, C. W. 2005, ApJL, 635, L181

de Kármán, T., \& Howarth, L. 1938, RSPSA, 164, 192

Donzis, D. A., Yeung, P. K., \& Sreenivasan, K. R. 2008, PhFl, 20, 045108

Galtier, S., Nazarenko, S. V., Newell, A. C., \& Pouquet, A. 2000, JPIPh, 63, 447

Galtier, S., Nazarenko, S. V., Newell, A. C., \& Pouquet, A. 2002, ApJL, 564 , L49

Gogoberidze, G. 2007, PhP1, 14, 022304

Goldreich, P., \& Sridhar, S. 1995, ApJ, 438, 763

Grappin, R. 1986, PhFl, 29, 2433

Horbury, T. S., Forman, M., \& Oughton, S. 2008, PhRvL, 101, 175005

Iroshnikov, R. S. 1963, AZh, 40, 742, [SvA, 7, 566 (1964)]

Kadomtsev, B. B., \& Pogutse, O. P. 1974, JETP, 38, 283, [ZhETF, 65, 575 (1973)]

Kraichnan, R. H. 1965, PhFl, 8, 1385

Leamon, R. J., Matthaeus, W. H., Smith, C. W., et al. 2000, ApJ, 537, 1054

Lee, E., Brachet, M. E., Pouquet, A., Mininni, P. D., \& Rosenberg, D. 2010, PhRvE, 81, 016318

MacBride, B. T., Forman, M. A., \& Smith, C. W. 2005, in Proc. Solar Wind 11: Soho 16 "Connecting Sun and Heliosphere,", ed. B. Fleck, T. Zurbuchen, \& H. Lacoste (ESA SP-592; Noordwijk: European Space Agency), 613

MacBride, B. T., Smith, C. W., \& Forman, M. A. 2008, ApJ, 679, 1644
Maron, J., \& Goldreich, P. 2001, ApJ, 554, 1175

Matthaeus, W. H., \& Goldstein, M. L. 1982, JGR, 87, 6011

Matthaeus, W. H., Goldstein, M. L., \& Roberts, D. A. 1990, JGR, 95, 20673

Matthaeus, W. H., Pouquet, A., Mininni, P. D., Dmitruk, P., \& Breech, B. 2008 , PhRvL, 100, 085003

Matthaeus, W. H., Servidio, S., Dmitruk, P., et al. 2012, ApJ, 750, 103

Matthaeus, W. H., \& Zhou, Y. 1989, PhFlB, 1, 1929

Milano, L. J., Matthaeus, W. H., Dmitruk, P., \& Montgomery, D. C. 2001, PhPl, 8,2673

Mininni, P. D. 2011, AnRFM, 43, 377

Monin, A. S., \& Yaglom, A. M. 1975, Statistical Fluid Mechanics, Vol. 2 (Cambridge, MA: MIT Press)

Montgomery, D. C. 1982, PhST, 2, 83

Oughton, S., Priest, E. R., \& Matthaeus, W. H. 1994, JFM, 280, 95

Oughton, S., Wan, M., Matthaeus, W. H., \& Servidio, S. 2013, in Thirteenth International Solar Wind Conference, Vol. CP (Melville, NY: AIP), in press

Podesta, J. J. 2009, ApJ, 698, 986

Politano, H., \& Pouquet, A. 1998a, GeoRL, 25, 273

Politano, H., \& Pouquet, A. 1998b, PhRvE, 57, R21

Pouquet, A., Brachet, M.-E., Lee, E., et al. 2011, in IAU Symp. 271, Vol. 6 , Astrophysical Dynamics: From Stars to Galaxies, ed. N. H. Brummell, A. S. Brun, M. S. Miesch, \& Y. Ponty (Cambridge: Cambridge Univ. Press), 304

Pouquet, A., Meneguzzi, M., \& Frisch, U. 1986, PhRvA, 33, 4266

Ren, Y., Almagri, A. F., Fiksel, G., et al. 2011, PhRvL, 107, 195002

Robinson, D. C., \& Rusbridge, M. G. 1971, PhFl, 14, 2499

Schekochihin, A. A., Cowley, S. C., Dorland, W., et al. 2009, ApJS, 182,310

Schekochihin, A. A., Nazarenko, S. V., \& Yousef, T. A. 2012, PhRvE, 85, 036406

Servidio, S., Matthaeus, W. H., \& Dmitruk, P. 2008, PhRvL, 100, 095005

Shebalin, J. V., Matthaeus, W. H., \& Montgomery, D. 1983, JPIPh, 29, 525

Smith, C. W. 1981, PhD thesis, College of William and Mary

Sorriso-Valvo, L., Marino, R., Carbone, V., et al. 2007, PhRvL, 99, 115001

Strauss, H. R. 1976, PhFl, 19, 134

Stribling, T., Matthaeus, W. H., \& Ghosh, S. 1994, JGR, 99, 2567

Wan, M., Oughton, S., Servidio, S., \& Matthaeus, W. H. 2010, PhP1, 17, 082308

Wan, M., Oughton, S., Servidio, S., \& Matthaeus, W. H. 2012, JFM, 697, 296

Zank, G. P., \& Matthaeus, W. H. 1992, JPIPh, 48, 85

Zhou, Y., \& Matthaeus, W. H. 2005, PhPl, 12, 056503

Zhou, Y., Matthaeus, W. H., \& Dmitruk, P. 2004, RvMP, 76, 1015

Zweben, S., Menyuk, C., \& Taylor, R. 1979, PhRvL, 42, 1270 